\title{
Patient "GRIT" Does Not Predict Pain and Opioid Consumption Following Total Knee Arthroplasty
}

\author{
Sydney Keller ${ }^{1}$, Sachin Seetharam ${ }^{1}$, \\ Mary Ziemba-Davis ${ }^{2}$, R. Michael Meneghini MD ${ }^{2,3}$ \\ ${ }^{1}$ Indiana University School of Medicine \\ ${ }^{2}$ Indiana University Health Physicians, Orthopaedics \\ ${ }^{3}$ Indiana University School of Medicine, Department of Orthopaedic Surgery \\ Background and Hypothesis: TKA results in severe postoperative pain and \\ differential needs for narcotics. Medical covariates of pain and opioid use \\ following TKA have been investigated, but the influence of personality has not. \\ GRIT consists of passion/consistency of interest in goals and perseverance of \\ effort. Perseverance has been associated with beneficial autonomic nervous \\ system activity. GRIT has been associated with independence in activities of \\ daily living, decreased depression/anxiety, and increased well-being in \\ chronically ill patients. We evaluated whether GRIT modulates pain and opioid \\ consumption following TKA, hypothesizing that higher GRIT scores would be \\ associated with lower pain and opioid consumption.
}

Project Methods: The GRIT survey was retrospectively administered to 254 consecutive patients with cemented TKAs performed between 2016 and 2018 by a single surgeon using the same pain protocols and implant. Results were paired with prospectively collected average pain score during the first 24 hours following PACU discharge, average pain during the remainder of hospital stay, final pain score prior to discharge, time to first opioid medication after PACU discharge, total opioid consumption in morphine equivalents (MEQs) during the first 24 hours after PACU, and average MEQs/day during the remainder of stay.

Results: The sample was $71 \%$ female with average age of $67.5 \pm 8.3$ years and average BMl of $33.7 \pm 7.2 \mathrm{~kg} / \mathrm{m}^{2}$. Coefficient $r$ for pain metrics and passion, perseverance, and GRIT ranged from -0.145 to $0.078(p \geq 0.146)$ with the exception of a small $(0.136)$, but significant $(p=0.032)$ correlation between final pain score and perseverance. $r$ for opioid metrics and passion, perseverance, and GRIT ranged from -0.039 to $0.087(p \geq 0.172)$.

Conclusion and Potential Impact: Mean GRIT scores ranged from 3.7-4.3 with a median range of 3.8-4.3, suggesting demand characteristics associated with the instrument. Post hoc statistical power was low $(\approx 35 \%)$ given $n$ and small effect sizes. It is hoped, however, that our study fosters investigation of individual characteristics and important clinical outcomes. 